\title{
An ultrasound program for the emergency department
}

\author{
Urbain Ip, MD
}

$I^{n}$ n February 1997, a proposal to develop diagnostic ultrasound (U/S) skills for emergency physicians at Surrey Memorial Hospital was presented to the Diagnostic Imaging (DI) Department and subsequently to the Medical Advisory Committee. To our surprise, this was accepted in principle, with no resistance. With the support of DI, maternity and emergency medicine developed a joint proposal to purchase a $\$ 160,000$ portable U/S machine. It happened so easily that I became convinced God is an emergency physician.

We purchased the machine late in 1997 and trained our EPs in April 1998, through a 2-day ultrasound course provided by faculty from the United States. Surgeons and obstetricians also attended the course, and additional training was provided locally using our own machine. DI and emergency medicine agreed that emergency physicians would only perform after-hours U/S exams, and that indications would be limited, initially, to detecting free fluid in the abdomen, looking for a suspected abdominal aortic aneurysm, identifying pericardial fluid, and determining whether a pregnancy is intrauterine (but not evaluating the pregnancy itself).

Just before program start-up, the Diagnostic Accreditation Program (DAP) sent us a letter expressing their disapproval of emergency physician U/S use. The letter stated that our proposed EP training guidelines fell short of existing DAP

standards for training obstetricians, cardiologists, surgeons and internists who use diagnostic ultrasound.

Although the DAP provides physician accreditation for various skills, including radiography, U/S interpretation, and electrocardiography, it is clear they do not understand the concept of using focused U/S examination as a clinical screening tool. While most Canadian EPs are not accredited to read electrocardiograms, computed tomography scans, or chest x-rays, they regularly make critical diagnostic and therapeutic decisions, such as whether to administer thrombolytic agents, based on their unaccredited interpretations. The DAP's concern may be that, if it accredits EPs to perform U/S, then these physicians might request a billing code, as other groups have. Not surprisingly, most DAP members are radiologists, and in my opinion, we do not need the approval of this group to proceed with bedside ED ultrasound initiatives.

The critical DAP letter made the hospital CEO and head of DI rather nervous; therefore, we elected to begin with a pilot study examining the safety, diagnostic accuracy, and time saved by goal-directed EP U/S exams. Since October 1998, we have performed 59 focused examinations on 48 patients. Our positive and negative predictive values are $100 \%$ and $99.5 \%$ respectively, and the study is ongoing.

Correspondence to: khung@interchange.ubc.ca

Head, Department of Emergency Medicine, Surrey Memorial Hospital, Surrey, BC

\section{A radiologist's perspective}

\author{
Derrick McPhee, MD
}

$\mathrm{F}_{\mathrm{s}}$ ocused abdominal sonography in trauma (FAST) has in many centres replaced the diagnostic peritoneal lavage (DPL) for the early assessment of acute blunt abdominal trauma. In many cases a negative FAST obviates the need for further imaging and intervention. In well-trained hands, it is a very specific and relatively sensitive test for the detection of hemoperitoneum and has the advantage over DPL of being noninvasive. However, the introduction of this exam has raised many contentious issues around indications for the study, as well as who should perform and interpret the study. Ironically, the question of who should perform the test has in many ways overshadowed the issue of whether it should be performed at all.

FAST ultrasound is clearly not appropriate for every

Queen Elizabeth II (QE II) Health Sciences Centre, and Dalhousie University, Halifax, NS 